ASTHMA

\title{
Treatment of ovalbumin-induced experimental allergic bronchitis in rats by inhaled inhibitor of secretory phospholipase $A_{2}$
}

\author{
D Shoseyov, H Bibi, S Offer, O Schwob, M Krimsky, M Kleiman, S Yedgar
}

Thorax 2005;60:747-753. doi: 10.1136/thx.2005.043695

See end of article for authors' affiliations

Correspondence to: Professor S Yedgar, Department of Biochemistry, Hebrew University-Hadassah Medical School,

Jerusalem, Israel 91120;

Yedgar@md.huji.ac.il

Received 14 March 2005

Accepted 25 June 2005

Published Online First

30 June 2005
Background: The pathophysiology of asthma involves the action of inflammatory/allergic lipid mediators formed following membrane phospholipid hydrolysis by phospholipase $A_{2}\left(P L A_{2}\right)$. Cysteinyl leukotrienes are considered potent inducers of bronchoconstriction and airway remodelling. Ovalbumin (OVA) induced bronchoconstriction in rats is associated with increased secretory $\mathrm{PLA}_{2}\left(\mathrm{sPLA}_{2}\right)$ activation and cysteinyl leukotriene production, together with suppression of cytosolic $\mathrm{PLA}_{2}$ and prostaglandin $\mathrm{E}_{2}$. These processes are reversed when the animals are pretreated systemically with an extracellular cell impermeable $\mathrm{SPLA}_{2}$ inhibitor which also suppresses the early allergic reaction to OVA challenge. In this study we examine the capacity of the $\mathrm{SPLA}_{2}$ inhibitor to ameliorate inflammatory and allergic manifestations (early and late bronchoconstriction) of OVA induced allergic bronchitis in rats when the inhibitor was administered by inhalation to confine it to the airways.

Methods: Rats sensitised with OVA were treated with the $\mathrm{SPLA}_{2}$ inhibitor hyaluronic acid-linked phosphatidyl ethanolamine (HyPE). The rats were divided into four groups ( $n=10$ per group): (1) naïve controls (no sensitisation/no treatment); (2) positive controls (sensitisation + challenge with OVA inhalation and subcutaneous injection of $1 \mathrm{ml}$ saline before each challenge; (3) sensitisation + challenge with OVA and HyPE inhalation before every challenge; and (4) sensitisation + challenge with OVA and treatment with subcutaneous dexamethasone $(300 \mu \mathrm{g})$ before each challenge as a conventional reference. Another group received no treatment with HyPE during the sensitisation process but only before or after challenge of already sensitised rats. Pulmonary function was assessed and changes in the histology of the airways, levels of cysteinyl leukotrienes in BAL fluid, and the production of nitric oxide (No) and tumour necrosis factor $\alpha$ (TNF $\alpha$ ) by BAL macrophages were determined.

Results: Inhalation of HyPE markedly suppressed OVA induced early and late asthmatic reactions as expressed by bronchoconstriction, airway remodelling (histology), cysteinyl leukotriene level in BAL fluid, and production of TNF $\alpha$ and NO by BAL macrophages. OVA induced bronchoconstriction in sensitised non-pretreated rats was also inhibited by inhalation of HyPE either before or after the challenge.

Conclusions: These findings confirm the pivotal role of $\mathrm{sPLA}_{2}$ in the pathophysiology of both the immediate allergic response and the inflammatory asthmatic process. Control of airway $\mathrm{SPLA}_{2}$ may be a new therapeutic approach to the treatment of asthma.
$\mathrm{T}$ he pathophysiology of asthma involves the action of inflammatory/allergic lipid mediators produced following hydrolysis of membrane phospholipids by phospholipase $\mathrm{A}_{2} \quad\left(\mathrm{PLA}_{2}\right)$ into lyso-phospholipids and arachidonic acid. Arachidonic acid is metabolised mainly via the lipoxygenase and cyclo-oxygenase pathways, producing leukotrienes (LTs) and prostaglandins (PGs), respectively. Cysteinyl LTs (CysLTs) in particular play an important role in the pathophysiology of asthma. ${ }^{1}$ Lyso-phospholipids induce white cell activation, histamine secretion from mast cells, and airway smooth muscle (ASM) proliferation. ${ }^{23}$

The $\mathrm{PLA}_{2}$ family consists of intracellular and secreted enzymes; ${ }^{4}$ the intracellular enzymes include the $\mathrm{Ca}^{2+}$ independent $\mathrm{iPLA}_{2}$ which is generally considered a housekeeping enzyme, and the cytosolic CPLA $\mathrm{C}_{2}$ enzyme which is specific to arachidonic acid carrying phospholipids and thus considered a major producer of arachidonic acid. Secretory $\mathrm{PLA}_{2}\left(\mathrm{sPLA}_{2}\right)$, secreted by activated leucocytes and other assaulted cells ${ }^{56}$ hydrolyse cell membrane phospholipids with no preference for fatty acid. sPLA ${ }_{2}$ also act as receptor ligands to induce production of cytokines (for example, tumour necrosis factor $\alpha(\mathrm{TNF} \alpha)$ and interleukin (IL) 6 and IL-8), ${ }^{78}$ mast cell survival, ${ }^{9}$ CD44 expression by eosinophils, ${ }^{8}$ and ASM cell proliferation. ${ }^{7}$ The $\mathrm{PLA}_{2}$ family is thus involved directly and indirectly in inducing inflammatory/allergic processes, especially sPLA 2 which is considered the "inflammatory enzyme" ${ }^{\prime}{ }^{6}$

Although the relative contributions of $\mathrm{CPLA}_{2}$ and $\mathrm{sPLA}_{2}$ differ between cells and tissues, it is generally accepted that both types take part and often act synergistically in producing pro-inflammatory lipid mediators. ${ }^{10}$ However, the airways seem to be an exception in which different eicosanoids play opposing roles: $\mathrm{PGE}_{2}$, generally considered a strong pro-inflammatory agent, is a potent bronchodilator and can inhibit ASM proliferation, ${ }^{11}{ }^{12}$ whereas CysLTs are strong bronchoconstrictors and facilitate airway remodelling. ${ }^{13}$ It has therefore been postulated that, unlike other organs, the lung is a privileged site for the beneficial actions of $\mathrm{PGE}_{2}{ }^{12}$ and in challenged airways LTs and PGs follow different routes. Accordingly, it has been reported that, in inflammatory cells, LTs are

\footnotetext{
Abbreviations: ASM, airway smooth muscle; CysLT, cysteinyl leukotriene; ExPLI, extracellular sPLA 2 inhibitor; GAG, glycosaminoglycans; HyPE, hyaluronic acid-linked phosphatidyl ethanolamine; IL, interleukin; LT, leukotriene; NO, nitric oxide; OVA, ovalbumin; PG, prostaglandin; PLA 2 , phospholipase $A_{2} ; T N F \alpha$, tumour necrosis factor $\alpha$
} 
produced from an SPLA $_{2}$ linked arachidonic acid pool while PGs are produced from a CPLA $_{2}$ linked pool of arachidonic acid, ${ }^{10} 14$ implying opposing roles for SPLA $_{2}$ and $\mathrm{CPLA}_{2}$ in asthma.

This hypothesis was strongly supported by our recent study with an experimental allergic bronchitis model in rats ${ }^{15}$ which showed that ovalbumin (OVA) induced bronchoconstriction is associated with an increase in SPLA $_{2}$ and CysLT levels and suppression of $\mathrm{CPLA}_{2}$ and $\mathrm{PGE}_{2}$ levels. Furthermore, these processes were reversed by treatment with an extracellular SPLA $_{2}$ inhibitor (ExPLI), suggesting a pivotal role for $\mathrm{sPLA}_{2}$ in the pathophysiology of allergy. In this study we have tested the potential of sPLA $_{2}$ inhibition in ameliorating experimental allergic bronchitis by examining the effect of ExPLI inhalation on the early and late bronchoconstriction reaction and airway inflammation and remodelling.

\section{METHODS}

\section{Induction of experimental allergic bronchitis}

Brown Norway rats were sensitised with OVA as previously described. ${ }^{15}$ Briefly, a subcutaneous injection of OVA with aluminium hydroxide and an intraperitoneal injection of Bordetella pertussis killed organisms were administered on day 0 . Two weeks later the rats were placed unrestrained in a 20 litre box connected to an ultrasonic nebuliser (LS 230 System, Villeneuve sur Lot, France) and subjected to repeated bronchial allergen challenge by inhalation of OVA $(1 \mathrm{mg} / \mathrm{ml}$ saline) for 5 minutes every other day to day 45 .

\section{Treatments}

The ExPLI used was hyaluronic acid-linked phosphatidyl ethanolamine (HyPE) which was synthesised in the laboratory of $\mathrm{S}$ Yedgar $^{16}$ by conjugating hyaluronic acid (HA, Gideon Richter, Budapest, Hungary) with dipalmitoyl phosphatidyl ethanolamine (PE, Sigma, St Louis, MI) to form an amide bond between the PE amino group and the HA carboxylic group, and was characterised by NMR spectroscopy. ${ }^{16} 17$

Two treatment modes were applied:

- HyPE inhalation during the induction of allergic bronchitis to examine the effect of ExPLI on sensitisation (prevention). Four treatment groups (each of 10 rats) were used:

- naïve control $(-/-)$ : no sensitisation/no treatment;

- positive control (OVA/OVA): sensitisation + challenge with OVA inhalation and subcutaneous injection of $1 \mathrm{ml}$ saline before each challenge;

- sensitisation + challenge with OVA and HyPE inhalation before every challenge (OVA/HyPE);

- sensitisation + challenge with OVA and treatment with subcutaneous dexamethasone $(300 \mu \mathrm{g})$ before each challenge (OVA/Dx) as a conventional reference.

- No treatment with HyPE during the sensitisation process but only before or after challenge of already sensitised rats.

Five $\mathrm{ml}$ HyPE solution ( $\mathrm{lmg} / \mathrm{ml}$ saline) was sprayed into the $20 \mathrm{l}$ cage, thus diluting the HyPE to $0.25 \mu \mathrm{g} / \mathrm{ml}$ aerosol, and the rats inhaled the aerosol for 5 minutes. The respiratory rate of the rats was 120 breaths/min with a mean tidal volume of $1 \mathrm{ml}$, giving a ventilation rate of $120 \mathrm{ml} / \mathrm{min}$. If all the inhaled HyPE was absorbed in 5 minutes $(600 \mathrm{ml})$ the maximal HyPE absorbed would be $150 \mu \mathrm{g}(3 \mathrm{nmol})$ in each treatment. Each group was treated and challenged every other day from day 14 until day 45 .

\section{Assessment of pulmonary function}

Unrestrained conscious rats were placed in a whole body plethysmograph (Buxco Electronics, Troy, NY, USA) to measure flow derived pulmonary function (Penh) as described elsewhere. ${ }^{15} 18$ Bronchoconstriction was assessed on day 20 by measuring Penh before and 5 minutes and 8 hours after OVA challenge, corresponding to the early and late asthmatic reaction, respectively.

\section{Histological examination}

On day 45 the rats were anaesthetised with an intraperitoneal injection of sodium pentobarbital, sacrificed by bleeding through the abdominal aorta, tracheotomised and cannulated through the trachea. Bronchoalveolar lavage (BAL) fluid was collected by repeated washing of the lungs with $5 \mathrm{ml}$ saline to a total of $50 \mathrm{ml}$. The lungs were removed, inflated, fixed with $4 \%$ formaldehyde under a pressure of $20 \mathrm{~cm} \mathrm{H}_{2} \mathrm{O}$, and embedded in paraffin. The fixed lungs were sliced longitudinally and embedded in paraffin. Histological sections $3 \mu \mathrm{m}$ thick were cut and stained with (1) haematoxylin and eosin for assessment of interstitial and peribronchial inflammation and of ASM thickening; (2) Tri-chrome for assessment of subepithelial fibrosis (basal membrane thickness); and (3) Periodic acid Schiff (PAS) for examination of epithelial cell mucus metaplasia.

Histological morphometry was performed using the computer program ImageJ (NIH, Bethesda, MD, USA) on three randomly selected slides from each lung. Quantification of peribronchial cellular infiltrate in airway tissue was determined by counting the numbers of inflammatory cells in the region $50 \mu \mathrm{m}$ beneath the epithelium of the airway in haematoxylin and eosin stained sections and expressed as the number of cells per mm airway basal lamina length which was measured by tracing the basal lamina in calibrated digital images. ASM and basal membrane thickening were determined as previously described.$^{18}$ Briefly, the area of ASM cells or the basal membrane thickness was normalised to the
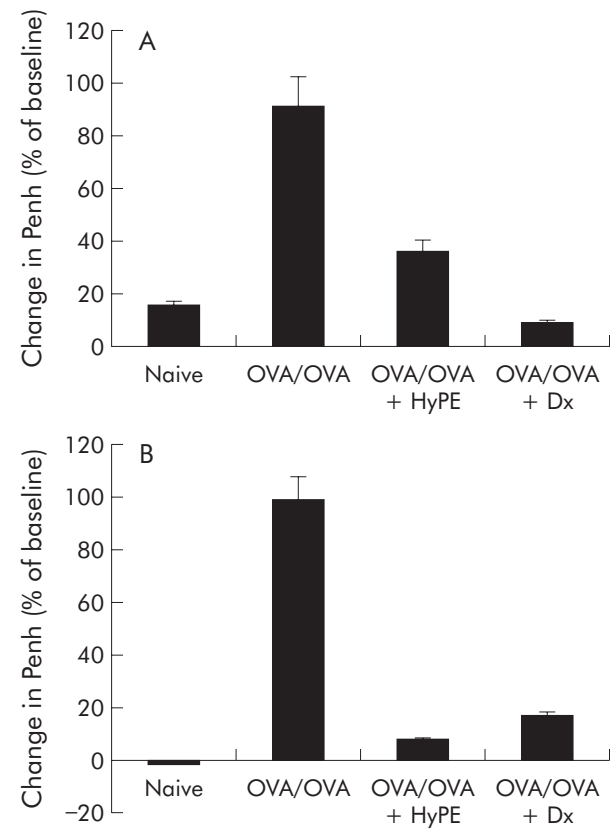

Figure 1 Effect of HyPE inhalation on (A) early and (B) late bronchoconstrictive reactions induced by ovalbumin (OVA) inhalation. Bronchoconstriction, expressed as the percentage change in Penh, ${ }^{16}$ was induced in OVA sensitised rats by inhalation of OVA and measured before, 5 minutes after, and 8 hours after allergen challenge. Data shown are mean (SE) values for 10 rats. $p<0.01$ between OVA/OVA and the other groups for both early and late reactions. 
square of the basement membrane length $\left(L_{\mathrm{bm}}{ }^{2}, \mu \mathrm{m}^{2}\right)$ to correct for differences in airway size. Mucus metaplasia was determined by the percentage of PAS positive cells in the total airway epithelial cells. Only large $\left(>2000 \mu \mathrm{m} L_{\mathrm{bm}}\right)$ and medium size airways $\left(1000-2000 \mu \mathrm{m} L_{\mathrm{bm}}\right)$ were selected as it has been found that the most significant pathological changes occur in these airways with less significant changes in the small airways. ${ }^{19}$ Airways with a ratio of maximum to minimum internal diameter of $\geqslant 2$ were considered to be cut tangentially and were not measured. The internal diameters were measured from the basement membrane on one side to the basement membrane on the opposite side of the airway.

\section{Biochemical markers}

CysLTs $\left(\mathrm{LTC}_{4}, \mathrm{LTD}_{4}\right.$ and $\left.\mathrm{LTE}_{4}\right)$ were determined by BAL enzyme immunoassay using a commercial kit (Amersham, $\mathrm{UK}) .{ }^{15}$

To measure production of nitric oxide (NO) and tumour necrosis factor $\alpha(\mathrm{TNF} \alpha)$ by macrophages, BAL macrophages were isolated and cultivated in DMEM $+10 \%$ serum for 2 hours, after which non-adherent cells were removed. The adherent cells were recultivated in DMEM $+10 \%$ serum $\left(10^{6}\right.$ cells/well) for 48 hours. The culture medium was then collected for determination of NO using the Gries method for measuring the corresponding nitrite, and TNF $\alpha$ using a radioimmunoassay kit (Amersham, UK).

\section{Analysis of data}

Statistical analysis was performed using statistical software (GB-STAT, Dynamic Microsystem, Silver Spring, MD, USA). Analysis of variance (ANOVA) was used to assess significant differences between treatment groups. The Tukey test was used to compare each of the treatment groups. A p value of $<0.05$ was considered significant.

\section{RESULTS}

\section{Effect of HyPE inhalation on respiratory function in rats with OVA induced experimental allergic bronchitis}

To examine the effect of ExPLI on sensitisation of rats by OVA, the rats inhaled HyPE before every challenge as described above. In parallel, another group was given a subcutaneous injection of dexamethasone (Dx). As shown in fig 1 , inhalation of HyPE was effective in reducing bronchoconstriction in both the early and late reactions.

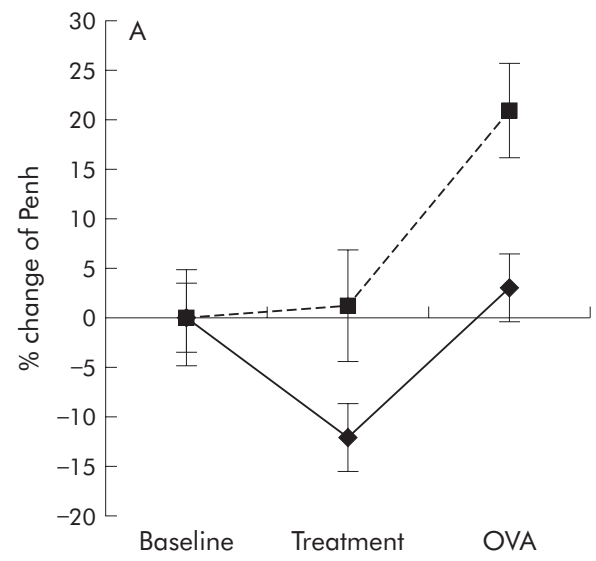

To examine the ability of inhaled HyPE to counteract OVA induced bronchoconstriction in untreated allergic reaction, rats sensitised with OVA but not pretreated with HyPE inhaled HyPE only once, either before or after challenge with OVA. Figure 2 shows that HyPE decreased OVA induced bronchoconstriction when inhaled either before or after the challenge.

Prevention of airway remodelling by HyPE inhalation To examine the effect of HyPE inhalation on allergic bronchial inflammation, at the end of the experiment (30 days after treatment and repeated challenge) the rats were sacrificed and tracheotomised, BAL fluid was collected, and lung tissues were processed for histological examination.

As shown in fig 3, peribronchiolar and perivascular cell infiltrates were composed principally of eosinophils and lymphocytes. The eosinophils were distributed in a circumferential manner around the bronchioles and vessels while the lymphocytes were either similarly distributed or arranged in primary follicles (lymphoid follicles without germinal centres). Reduced inflammatory cell infiltration and airway wall thickening was seen in HyPE treated rats. Table 1 shows that inhalation of HyPE reduced peribronchial cellularity, mucus metaplasia of respiratory epithelial cells, ASM thickening, and basal membrane thickening essentially to the level of the naïve rats.

\section{Effect of HyPE inhalation on biochemical markers}

Among the lipid mediators involved in asthma pathophysiology, CysLTs are considered key mediators of bronchoconstriction and the inhibition of their action is currently the target of several drugs (montelukast, zileuton) used for the treatment of asthma. ${ }^{20}$ As the PLA 2 inhibitor controls lipid mediator production, it was of particular interest to determine its effect on CysLT production in OVA sensitised rats. CysLT levels in the BAL fluid of the different groups were determined by ELISA. As shown in fig 4, CysLT production was markedly enhanced in sensitised rats but was reduced to the normal range by inhalation of HyPE.

Nitric oxide production is a characteristic marker of inflammation in asthma which correlates with disease severity, although it might have dual effects on mast cells.. ${ }^{21}$ Its production has been reported to be linked to PLA produced lipid mediators ${ }^{22}$ and was found to be inhibited by ExPLIs in lipopolysaccharide stimulated cells ${ }^{23}$ and animals. ${ }^{24}$

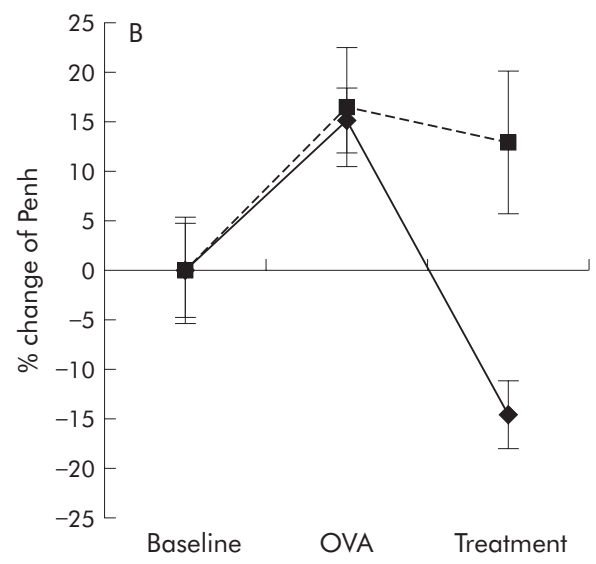

Figure 2 Immediate effect of HyPE inhalation on ovalbumin (OVA) induced bronchoconstriction in OVA sensitised rats. (A) Prevention of bronchoconstriction: OVA sensitised rats (not treated with HyPE during sensitisation) were subjected to Penh determination at baseline, 5 minutes after inhalation of HyPE (diamonds) or saline (squares), and 5 minutes after OVA inhalation. (B) Relief of bronchoconstriction: OVA sensitised rats (not treated with HyPE during sensitisation) were subjected to Penh determination at baseline, 5 minutes after inhalation of OVA, and 5 minutes after inhalation of HyPE (diamonds) or saline (squares). Data points are mean (SE) for five rats. In (A) $p<0.05$ between HyPE and saline treated rats and OVA+HyPE treated rats. In (B) $p<0.01$ between HyPE and saline treated rats. 
A
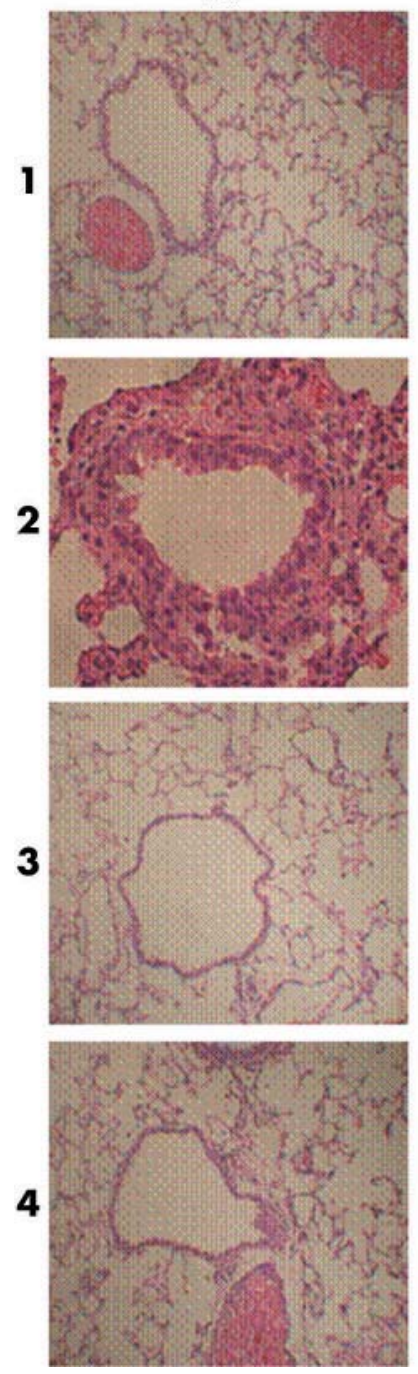

B
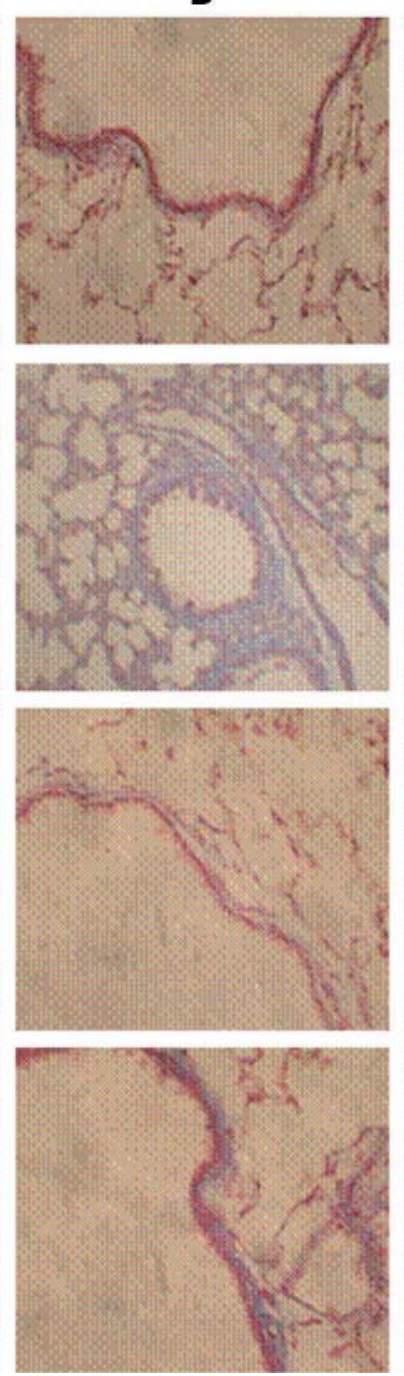

C
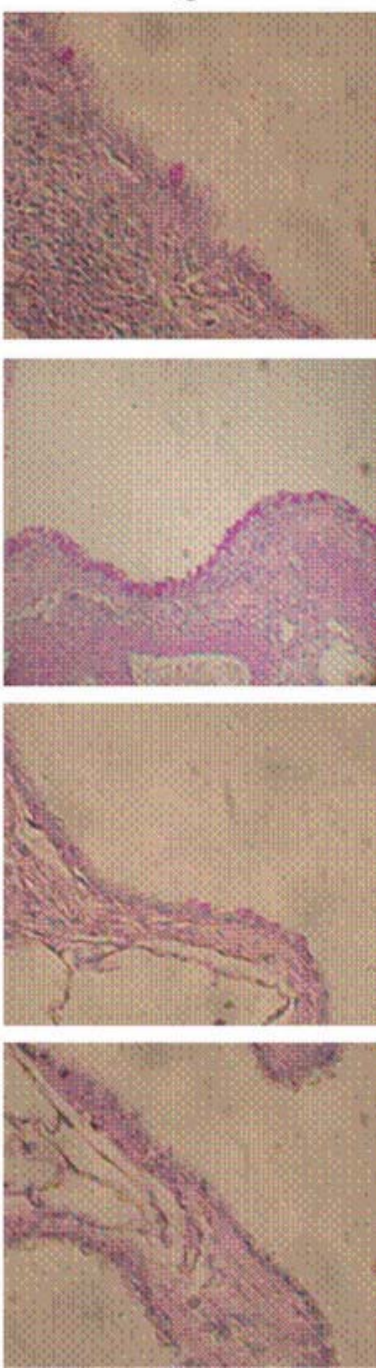

Figure 3 Effect of HyPE inhalation on structural changes in airways (airway remodelling) of OVA sensitised rats. Rats were subjected to OVA inhalation every other day for 30 days. Rats treated with HyPE inhaled HyPE aerosol for 5 minutes before each allergen inhalation. The rats were sacrificed on day 45. (A) Haematoxylin-eosin stain for detection of inflammatory cell infiltration and changes in airway smooth muscle cell (ASM) thickness. (B) Mason-Trichrom stain of connective tissue (collagen) for detection of changes in basal membrane thickness. (C) Periodic Acid Schiff (PAS) stain for detection of mucus metaplasia of respiratory epithelial cells. 1, 2, 3 and 4 indicate tissues of naiive, OVA/OVA, HyPE treated, and dexamethasone treated rats, respectively.

Table 1 Effect of HyPE on remodelling of OVA sensitised rat airways: histological morphometry

\begin{tabular}{lllll}
\hline & Naïv & Asthma & Asthma + HyPE & Asthma + Dx \\
\hline $\begin{array}{l}\text { (I) Inflammatory cell } \\
\text { infiltration }\end{array}$ & $1.16(0.11)$ & $11.41(1.81)$ & $1.44(0.21)$ & $1.41(0.16)$ \\
$\begin{array}{l}\text { (II) Airway smooth muscle } \\
\text { thickening }\end{array}$ & $3.96(0.39)$ & $40.20(4.78)$ & $5.03(0.65)$ & $7.10(0.96)$ \\
$\begin{array}{l}\text { (III) Basement membrane } \\
\text { thickening }\end{array}$ & $0.76(0.20)$ & $8.22(1.20)$ & $0.70(0.09)$ & $0.69(0.08)$ \\
(IV) Mucus metaplasia & $12.0(1.3)$ & $67.0(3.2)$ & $13.0(4.1)$ & $11.0(3.5)$ \\
\hline
\end{tabular}

1, Infiltration of inflammatory cells into the space between the intima and adventitia of the bronchi, expressed as number of inflammatory cells per mm of basal membrane. II, Airway smooth muscle (ASM) thickening expressed as width of ASM per length of basal membrane $(\mu \mathrm{m} / \mathrm{mm})$. III, Thickening of basal membrane expressed as width ( $\mu \mathrm{m})$ per length $(\mathrm{mm})$ of basement membrane. IV, Mucus metaplasia of respiratory epithelial cells expressed as the percentage of PAS positive cells from total epithelial cells.

Data are mean (SE) values for 10 rats. For all parameters, statistical analysis showed a significant difference $(p<0.001)$ between non-treated asthmatic rats and the other groups, and no significant difference between HyPE treated and naive or dexamethasone (Dx) treated rats. 


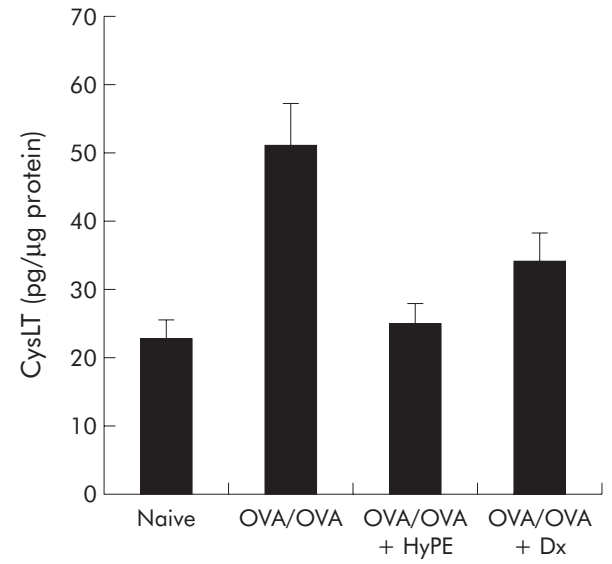

Figure 4 Effect of HyPE inhalation on cysteinyl leukotriene (LTC ${ }_{4}, \mathrm{LTD}_{4}$ and $\mathrm{LTE}_{4}$ ) levels in BAL fluid of ovalbumin (OVA) sensitised rats. BAL fluid was collected upon sacrifice and CysLT levels were determined by enzyme immunoassay. Data are mean (SE) values for 10 rats. A significant difference was seen between OVA/OVA and HyPE treated rats $(p<0.01)$. No significant differences were seen between HyPE treated and naive or dexamethasone (Dx) treated rats.

In line with these findings, we determined the effect of ExPLI on NO production by cultured macrophages collected from BAL fluid of the different groups following their in vivo treatment without further treatment of the cultured cells. Figure 5 shows that NO production was considerably enhanced in BAL macrophages of sensitised rats (OVA/ OVA) compared with naïve $(-/-)$ rats, but NO production by macrophages from sensitised rats treated with HyPE inhalation was below the level of the naïve rats.

A prominent characteristic marker of inflammatory conditions is TNF $\alpha$ which is at the top of other pro-inflammatory cytokine cascade. The increase in its level correlates with the severity of inflammation in asthma, and its inhibition has been proposed for the treatment of severe asthma. ${ }^{25}$ In previous studies the ExPLIs were found effective in suppressing endotoxin stimulated production of $\mathrm{TNF} \alpha$ and other cytokines in animals and cell cultures. ${ }^{24}{ }^{26}$ Similarly, we found that TNF $\alpha$ production by macrophages from BAL fluid of sensitised rats was markedly enhanced, but inhalation of HyPE reduced it to approximately the level of the naïve rats (fig 6).

\section{DISCUSSION}

Inhibition of PLA 2 activity has been considered a promising therapeutic strategy in the treatment of inflammatory/allergic diseases as it can suppress the production of several classes of pathogenic lipid mediators (PGs, LTs, platelet aggregating factor, lysophospholipids). A number of PLA 2 inhibitors have been proposed for this purpose. ${ }^{27}$ However, PLA 2 inhibitors that enter the cell might interfere with the vital phospholipase metabolism and might impair the cell viability. In addition, as previously discussed, ${ }^{6}$ in inflammatory conditions a number of sPLAs are involved in cell lysis and lipid mediator production, and inhibiting only one or part of them has limited or no efficacy. Therefore, as has long been proposed, ${ }^{28}$ there is a clear advantage to cell impermeable $\mathrm{PLA}_{2}$ inhibitors that protect the cell membrane from the action of sPLA $_{2} \mathrm{~s}$ but are not internalised. ${ }^{6}$ These requirements are provided by the ExPLIs which are composed of a lipidic $\mathrm{PLA}_{2}$ inhibitor conjugated with a polymeric carrier. ${ }^{17}{ }^{29}$ While the lipidic component is incorporated into the cell membrane, its internalisation is prevented by the polymeric carrier. These kind of lipid conjugates have been shown to protect membranes from diverse types of $\mathrm{sPLA}_{2} \mathrm{~S}^{29}{ }^{30}$ and ameliorate

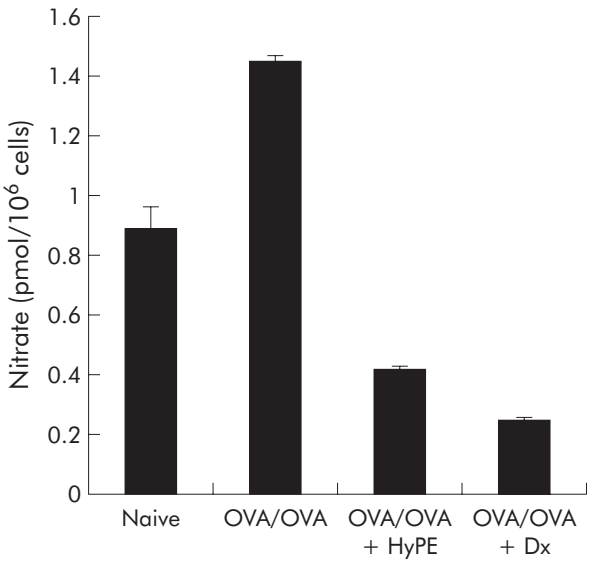

Figure 5 Effect of HyPE inhalation on nitric oxide (NO) production by macrophages collected from BAL fluid of ovalbumin (OVA) sensitised rats. Macrophages collected from BAL fluid of the different groups were cultured without further treatment with HyPE or dexamethasone (Dx) and NO production was determined by the corresponding nitrate level in the culture medium. Data are mean (SE) values for 10 rats. The NO level was reduced compared with OVA/OVA and naive rats by both HyPE $(p<0.001$ and $p<0.001$, respectively) and $D x(p<0.001$ and $p<0.001$, respectively).

different $\mathrm{sPLA}_{2}$ related inflammatory conditions including endotoxin induced sepsis in rats, ${ }^{24}$ allergic experimental encephalomyelitis in rats and mice, ${ }^{23}$ and TNBS induced colitis in rats. ${ }^{31}$

We have previously shown that sensitisation of rats with OVA is associated with an increase in $\mathrm{SPLA}_{2}$ and CysLT production together with suppression of $\mathrm{CPLA}_{2}$ and $\mathrm{PGE}_{2}$ production. These processes were reversed by systemic (subcutaneous) treatment with the cell impermeable PLA $_{2}$ inhibitor HyPE which also suppressed the early response (bronchoconstriction) to challenge with OVA. ${ }^{15}$ The present study shows that effective treatment of asthma can be obtained by application aimed at confining the treatment to the airway system. Inhalation of HyPE by asthmatic rats suppressed OVA induced sensitisation and airway inflammation (fig 1, table 1) while at the same time blocking CysLT

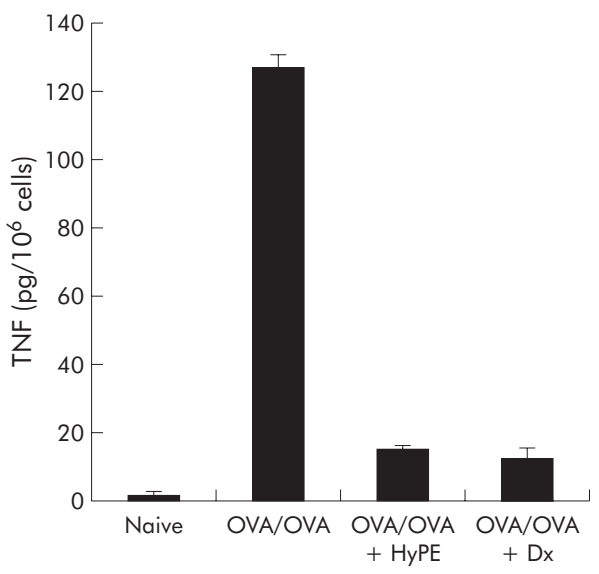

Figure 6 Effect of HyPE inhalation on tumour necrosis factor $\alpha$ (TNF $\alpha$ ) production by macrophages collected from BAL fluid of ovalbumin (OVA) sensitised rats. Macrophages collected from BAL fluid of the different groups were cultured without further treatment with HyPE or dexamethasone (Dx) and secretion of TNF $\alpha$ into the culture medium was determined. Data are mean (SE) values for 10 rats. $p<0.001$ for difference between OVA/OVA and HyPE treated rats. There was no significant difference between HyPE treated, naïve, and Dx treated rats. 
production (fig 4), and also reversed OVA induced bronchoconstriction in already sensitised rats (fig 2). Together, the present and preceding studies provide strong evidence for the pivotal role of $\mathrm{sPLA}_{2}$ in the pathophysiology of both the immediate allergic response and the inflammatory process.

Previous studies have pointed out the involvement of $\mathrm{PLA}_{2}$ in $\mathrm{NO}$ production, showing that exogenous $\mathrm{PLA}_{2}$ induces iNO synthase (iNOS) expression and NO generation by alveolar macrophages, a phenomenon that contributes to lung injury. ${ }^{22}$ In accordance with this we have found that treatment of rat glial cells with ExPLIs inhibits LPS induced production of $\mathrm{NO}$ concomitantly with $\mathrm{PGE}_{2}$ production and $\mathrm{PLA}_{2}$ secretion as measured by its lipolytic activity. ${ }^{23}$ In a study of the effect of ExPLI on sepsis in rats, we found that treatment with HyPE suppressed expression of iNOS and SPLA $_{2}$-IIA mRNA in lung and kidney of rats with endotoxin induced sepsis. ${ }^{24}$ The present study provides further evidence for the relationship between $\mathrm{NO}$ production and $\mathrm{PLA}_{2}$ activity, showing that treatment with the extracellular PLA 2 inhibitor suppressed the capacity of BAL macrophages to produce NO (fig 5).

The marked reduction in the ability of BAL macrophages to produce $\mathrm{TNF} \alpha$ following in vivo treatment of asthmatic rats with HyPE, without further treatment of the cultured cells (fig 6), may be attributed to $\mathrm{PLA}_{2}$ activity. However, the

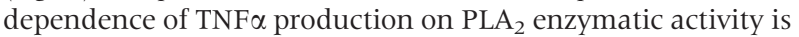
unclear. In a previous study from our laboratory it was found that ExPLIs inhibited lipopolysccharide induced production of different cytokines in cultured endothelial cells, ${ }^{26}$ which would suggest a relation to $\mathrm{PLA}_{2}$ activity. However, in that study we could not prove a dependence of cytokine production on $\mathrm{PLA}_{2}$ lipolytic activity. On the other hand, treatment with ExPLIs inhibited lipopolysaccharide induced activation of NFK-B which mediates TNF $\alpha$ production in cultured endothelia cells ${ }^{26}$ as well as $\mathrm{TNF} \alpha$ production in septic rats. ${ }^{24}$ As discussed above, sPLA 2 enzymes can induce cytokine production via a receptor mediated process which is independent of their enzymatic activity. ${ }^{7}$ It is thus possible that the effect of ExPLIs on cytokine production is due to suppression of $\mathrm{PLA}_{2}$ action as a receptor ligand rather than its enzymatic activity.

The lipolysis independent effects of the ExPLIs may be attributed to the fact that they are membrane anchored glycosaminoglycans (GAGs) or GAG-like molecules. Cell surface GAGs are known to protect the cell from exogenous damaging agents such as endotoxins, reactive oxygen species, degradation enzymes (for example, heparanase), and cytokines. ${ }^{32}{ }^{33}$ A number of studies have shown that the action of sPLA $_{2}$ on the cell membrane is inhibited by cell surface GAGs such as heparin ${ }^{34}$ and hyaluronic acid. ${ }^{29}{ }^{35}$ In a study of the synergistic action of hydrogen peroxide and sPLA $\mathrm{P}_{2}$ we have previously observed that only after the cell surface GAGs are degraded by hydrogen peroxide is the cell membrane rendered accessible to lysis by exogenous SPLA $_{2}{ }^{32}$ Although the relationship between GAG function and the action of inflammatory mediators is not unequivocally clear, it is well documented that GAG stripping exposes cells and tissues to damaging agents. Accordingly, it has been proposed that enrichment of cell surface GAGs would assist in protecting the cell from infection and inflammatory/allergic assaults. ${ }^{36}$ These requirements are fulfilled by the ExPLIs, which are composed of $\mathrm{PLA}_{2}$ inhibiting molecules such as $\mathrm{N}$-derivatised phosphatidyl ethanolamine to polymeric carriers (for example, hyaluronic acid or chondroitin sulfates). Because of their structure, these lipid conjugates exhibit a multiple effect in cell protection. The lipid moiety, which incorporates into the cell membrane, suppresses membrane phospholipase hydrolysis by endogenous $\mathrm{SPA}_{2}$ and the subsequent production of lipid mediators ${ }^{23}{ }^{29-31}$ while, at the same time, the polymeric carrier mimics the cell surface GAG in protecting cells from exogenous $\mathrm{SPLA}_{2}$ and other inflammatory agents such as reactive oxygen. ${ }^{32}{ }^{35}$ The soundness of this concept has been demonstrated in several in vivo studies, as cited above. ${ }^{23} 2431$ Of the different GAGs, hyaluronic acid is of particular relevance to the present study since it specifically binds to CD44 on lymphocytes and eosinophils and activates them. Accordingly, binding of hyaluronic acid to lymphocyte CD44 prevents their rolling and subsequent extravasation to inflammatory sites. ${ }^{37}$ This makes HyPE-like lipid conjugates particularly suitable for the treatment of asthma, and this indeed is shown by the findings of the present study.

In conclusion, the results of this study suggest a novel therapeutic approach to the treatment of allergic/inflammatory diseases and present the lipid conjugates-which concomitantly control $\mathrm{PLA}_{2}$ action and mimic cell surface GAG function-as a prototype of potential multifunctional non-steroidal inhaled drugs for the comprehensive treatment of asthma.

\section{Authors' affiliations}

D Shoseyov, Pediatric Department, Hadassah University Hospital, Mount Scopus, Jerusalem, Israel

H Bibi, Pediatric Pulmonology Clinic, Barzilai Hospital, Ashkelon, Israel S Offer, Institute of Biochemistry, Faculty of Agriculture, Hebrew University, Jerusalem, Rehovot, Israel

O Schwob, M Krimsky, M Kleiman, S Yedgar, Department of Biochemistry, Hebrew University-Hadassah Medical School, Jerusalem, Israel

This study was supported by a grant to $S$ Yedgar from the Walter and Greta Stiel Chair and a grant to D Shoseyov and S Yedgar from the Israel Ministry of Health.

Competing interests: none declared.

\section{REFERENCES}

1 Wenzel SE. Arachidonic acid metabolites: mediators of inflammation in asthma. Pharmacotherapy 1997;17:3-12S.

2 Toews ML, Ediger TL, Romberger DJ, et al. Lysophosphatidic acid in airway function and disease. Biochim Biophys Acta 2002;1582:240-50.

3 Lloret S, Moreno JJ. $\mathrm{Ca}^{2+}$ influx, phosphoinositide hydrolysis, and histamine release induced by lysophosphatidylserine in mast cells. J Cell Physiol 1995; 165:89-95.

4 Diaz BL, Arm JP. Phospholipase A(2). Prostagland Leukot Essential Fatty Acids 2003:69:87-97.

5 Vadas P, Browning J, Edelson J, et al. Extracellular phospholipase $\mathrm{A}_{2}$ expression and inflammation: the relationship with associated disease states. $J$ Lipid Mediat 1993;8:1-30

6 Yedgar S, Lichtenberg D, Schnitzer E. Inhibition of phospholipase $A_{2}$ as a therapeutic target. Biochim Biophys Acta 2000;1488:182-7.

7 Hanasaki K, Arita H. Phospholipiase $A_{2}$ receptor: a regulator of biological functions of secretory phospholipase $\mathrm{A}_{2}$. Prostagland Other Lipid Mediat 2002;68-69:71-82

8 Triggiani M, Granata F, Balestrieri B, et al. Secretory phospholipases $A_{2}$ activate selective functions in human eosinophils. J Immunol 2003; 170:3279-88

9 Fonteh AN, Marion CR, Barham BJ, et al. Enhancement of mast cell survival: a novel function of some secretory phospholipase $A_{2}$ isotypes. J Immunol $2001 ; 167: 4161-71$

10 Capper EA, Marshall LA. Mammalian phospholipases A(2): mediators of inflammation, proliferation and apoptosis. Prog Lipid Res 2001;40:167-97.

11 Pavord ID, Tattersfield AE. Bronchoprotective role for endogenous prostaglandin $\mathrm{E}_{2}$. Lancet 1995;345:436-8.

12 Vancheri C, Mastruzzo C, Sortino MA, et al. The lung as a privileged site for the beneficial actions of PGE 2 . Trends Immunol 2004;25:40-6.

13 Holgate ST, Peters-Golden M, Panettieri RA, et al. Roles of cysteinyl leukotrienes in airway inflammation, smooth muscle function, and remodeling. J Allergy Clin Immunol 2003;111(1 Suppl):S18-36.

14 Munoz NM, Kim YJ, Meliton AY, et al. Human group V phospholipase $A_{2}$ induces group IVA phospholipase $\mathrm{A}_{2}$-independent cysteinyl leukotriene synthesis in human eosinophils. J Biol Chem 2003;278:38813-20.

15 Offer S, Yedgar S, Schwob O, et al. Negative feedback between secretory and cytosolic phospholipase $\mathrm{A}_{2}$ and their opposing roles in ovalbumininduced bronchoconstriction in rats. Am J Physiol Lung Cell Mol Physiol 2005;288:L523-9.

16 Yedgar S. Use of lipid conjugates in the treatment of disease. Patent Reference \# US-2005-0079211-A1, 2005.

17 Schnitzer E, Dagan A, Krimsky M, et al. Interaction of hyaluronic acid-linked phosphatidylethanol-amine (HyPE) with LDL and its effect on the susceptibility of LDL lipids to oxidation. Chem Phys Lipids 2000;104:149-60. 
18 Hamelmann E, Schwarze G, Takeda K, et al. Noninvasive measurements of airway responsiveness in allergic mice using barometry plethysmography. Am J Respir Crit Care Med 1997;156:766-75.

19 Panettieri RA Jr, Murray RK, Eszterhas AJ, et al. Repeated allergen inhalations induce DNA synthesis in airway smooth muscle and epithelial cells in vivo. Am J Physiol Lung Cell Mol Physiol 1998;274:L417-24.

20 ZYFLO filmtab. In: Physicians' desk reference, 52nd ed. Montvale, NJ: Medical Economics, 1998:474-6.

21 Coleman JW. Nitric oxide: a regulator of mast cell activation and mast cellmediated inflammation. Clin Exp Immunol 2002;129:4-10.

22 Tsukahara $Y$, Morisaki T, Horita $Y$, et al. Phospholipase $A_{2}$ mediates nitric oxide production by alveolar macrophages and acute lung injury in pancreatitis. Ann Surg 1999;229:385-92.

23 Pinto $\mathrm{F}$, Brenner $\mathrm{T}$, Dan $\mathrm{O}$, et al. Extracellular phospholipase $\mathrm{A}_{2}$ inhibitor suppress central nervous system inflammation. GLIA 2003:44:275-82.

24 Beck G Ch, Hermes WC, Yard BA, et al. Amelioration of endotoxin-induced sepsis in rats by membrane-anchored lipid conjugates. Crit Care Med 2003;31:2015-21.

25 Thomas PS. Tumor necrosis factor-alpha: the role of this multifunctional cytokine in asthma. Immunol Cell Biol 2001;79:132-40.

26 Beck G Ch, Yard BA, Schulte J, et al. Inhibition of LPS-induced chemokine production in human lung endothelial cells by lipid conjugates anchored to the membrane. Br J Pharmacol 2002;135:1665-74.

27 Balsinde J, Balboa MA, Insel PA, et al. Regulation and inhibition of phospholipase A $A_{2}$. Ann Rev Pharmacol Toxicol 1999;39:175-89.

28 Blackwell GJ, Flower RJ. Inhibition of phospholipase. BrMed Bull 1983;39:260-4.
29 Dan P, Dagan A, Krimsky M, et al. Inhibition of type I and type II phospholipase $A_{2}$ by phosphatidyl-ethanolamine linked to polymeric carriers. Biochemistry 1998;37:6199-204

30 Balsinde J, Balboa MA, Yedgar S, et al. Group V phospholipase $\mathrm{A}_{2}$-mediated oleic acid mobilization in lipopolysaccharide-stimulated P388D macrophages. J Biol Chem 2000;275:4783-6.

31 Krimsky M, Yedgar S, Aptekar L, et al. Amelioration of TNBS-induced colon inflammation in rats by phospholipase $\mathrm{A}_{2}$ inhibitor. Am J Physiol Gastrointest Liver Physiol 2003;285:G586-92.

32 Dan $\mathrm{P}$, Nitzan DW, Dagan $\mathrm{A}$, et al. $\mathrm{H}_{2} \mathrm{O}_{2}$ renders cells accessible to lysis by exogenous phospholipase $\mathrm{A}_{2}$ : a novel mechanism for cell damage in inflammatory processes. FEBS Lett 1996;383:75-8.

33 Proudfoot AEI, Power CA, Rommel C, et al. Strategies for chemokine antagonists as therapeutics. Semin Immunol 2003;15:57-65.

34 Diccianni MB, Mistry MJ, Hug K, et al. Inhibition of phospholipase $A_{2}$ by heparin. Biochim Biophys Acta 1990;1046:242-8.

35 Nitzan DW, Dan P, Yedgar S. The role of hyaluronic acid in protecting surface phospholipids from lysis by exogenous phospholipase $A_{2}$. Rheumatology $2001 \cdot 40: 336-40$.

36 Russell A-L. Glycoaminoglycan (GAG) deficiency in protective barrier as an underlying, primary cause of ulcerative colitis, Crohn's disease interstinal cystitis and possibly Reiter's syndrome. Medical Hypotheses 1999:52:297-301.

37 Katoh S, Matsumoto N, Kawakita K, et al. A role for CD44 in an antigeninduced murine model of pulmonary eosinophilia. J Clin lnvest 2003;111:1563-70.

\section{LUNG ALERT}

Race and risk of lung cancer

$\Delta$ Cote ML, Kardia SLR, Wenzlaff AS, et al. Risk of lung cancer among white and black relatives of individuals with earlyonset lung cancer. JAMA 2005;293:3036-42

$\mathrm{T}$

he investigators collected interview data on 692 patients with early onset lung cancer, 773 matched controls, and 7576 first degree relatives of cases and controls. Patients were diagnosed between 1990 and 2003 in Detroit, USA and all were aged less than 50 years at diagnosis. One third of the study population was black. Age, sex, race, history of COPD, pneumonia, and smoking pack years were included in the analysis.

The study found that smokers with a family history of early lung cancer had a higher risk of developing lung cancer with increasing age than smokers without a family history. The increase in risk occurred after the age of 60 and, by the age of 70, 17.1\% (SE 2.4\%) of white relatives and $25.1 \%$ (SE 5.8\%) of black relatives had a diagnosis of lung cancer. Relatives of black cases were at a statistically increased risk of lung cancer than white relatives, even after adjusting for other factors (OR 2.07, 95\% CI 1.29 to 3.32 ).

The results from this paper could be used to discuss risk with relatives of patients with early onset lung cancer. Smoking habits and a family history of lung cancer should be recorded for adults presenting with respiratory symptoms.

K Ward

Specialist Registrar, East Kent Hospitals NHS Trust, UK 